\title{
A Technique for Rapid Non-Suture Vascular Anastomosis
}

\author{
Fred Gentili, W.M. Lougheed, Sunao Yoshijima, \\ Hideki Hondo and Margot B. MacKay
}

\begin{abstract}
In an attempt to overcome some of the difficulties inherent in vascular anastomosis using standard suture methods, we have developed a technique that allows for rapid non-suture anastomosis of blood vessels. The technique has been used in over 400 anastomoses, including interposition vein grafts in rat, rabbit and dog vessels ranging in size from $0.7 \mathrm{~mm}$ to $3 \mathrm{~mm}$ in diameter. Animals have been followed for over 1 year postoperatively with serial angiography and histological evaluation including scanning electron microscopy. Stress studies have compared the biomechanical properties of both non-suture and suture anastomosis. The overall patency rate in non-suture end-to-end anastomoses using an interposition vein graft was $85 \%$ at 1 year follow up. Histological evaluation revealed a healing pattern that was temporally similar to that of the suture technique. Stress tests revealed no significant difference between suture and non-suture technique. These studies reveal that the non-suture technique compares favourably with the suture technique, and demonstrate the feasibility of applying this technique to man.
\end{abstract}

RÉSUMÉ: Une technique rapide d'anastomose vasculaire sans suture Dans le cadre d'une recherche visant à surmonter certaines des difficultés inhérentes à l'anastomose vasculaire par les méthodes de suture standards, nous avons développé une technique qui permet une anastomose rapide des vaisseaux sanguins sans utiliser de suture. Cette technique a été utilisée dans plus de 400 anastomoses vasculaires, incluant des greffes de veines par interposition chez le rat, le lapin et le chien, au niveau de vaisseaux dont le diamètre variait de 0.7 à $3 \mathrm{~mm}$. Les animaux ont été suivis sur une période de I ans après l'opération, au moyen d'angiographies sériées et d'une évaluation histologique incluant la microscopie électronique à balayage. Les propriétés biomécaniques des anastomoses sans suture et avec sutures ont été comparées par des études de stress. Le taux global de perméabilité dans les anastomoses bout-à-bout sans suture, utilisant une greffe veineuse d'interposition, était de $85 \%$ à l'examen de contrôle un an après la procédure. L'évaluation histologique a montré un pattern de guérison similaire dans le temps à celui des anastomoses par sutures. Des épreuves de stress n'ont révélé aucune différence significative entre les techniques avec et sans suture. Ces études démontrent que la technique sans suture se compare favorablement à la technique par suture et atteste de la possibilité d'appliquer cette technique chez l'humain.

Can. J. Neurol. Sci. 1987; 14:92-95

The introduction of the operating room microscope and the development of microsurgical techniques have led to significant advances in vascular surgery. Nevertheless, many problems remain. In cerebrovascular surgery, these include the technical difficulty of working with small caliber vessels in deeply confined areas, the prolonged occlusion time required, especially for multiple anastomoses, and the difficulty in providing adequate brain perfusion during the anastomosis. The patency rates for long vein grafts from neck to proximal intracranial vessels remain disappointing. These problems are, to a large extent, unavoidable with manual suture techniques currently employed for vascular anastomosis. In an attempt to overcome some of these disadvantages, we have over the past 7 years developed a non-suture technique for vascular anastomosis.
The technique allows rapid, precise, intima-to-intima anastomosis of both large and small caliber vessels with a marked reduction in operating time, and is applicable to both end-toend and end-to-side anastomosis. The purpose of the present report is to review the current status of this technique and discuss the feasibility of its use in man.

\section{Material and Methods}

The technique for non-suture anastomosis has been previously described.' The device for end-to-end anastomosis utilizes 2 components, a grooved cylinder of sterilizable. biocompatible material (Elgiloy) and a ring-shaped clip with opposed ends separable under spring tension. The bipartite 
device provides intima-to-intima contact using a lapped joint principle with no foreign material within the lumen. Specially developed instruments facilitate the use of the components of the device during anastomosis. Initially, careful measurements of both the external and internal diameters of the recipient, donor and, when employed, vein grafts, are taken and appropriately sized cylinders and clips chosen. One end of the vessel to be anastomosed is mounted on the cylinder by passing it through the lumen and cuffing the end back over the metal tube. A circumferential clip is then applied and released to secure the vessel (Figure 1). When a vein graft is utilized, the other end is treated in identical fashion. The cylinder, with 1 end of the vessel or vein graft mounted, is held with a cylinder holder and the second, or recipient end evaginated over the first to effect a lapped joint; the intima-to-intima seal is then completed with a second ring clip that fits in the remaining groove on the cylinder (Figure 2).

The device for non-suture end-to-side anastomosis is a modification of the end-to-end prosthesis and likewise consists of 2 components, a cylinder and a ring-shaped clip incorporating multiple spikes radiating from the base and having opposed ends separable under spring pressure. The donor vessel is initially mounted on the cylinder by threading it through the cylinder and cuffing the end back over the tube (Figure 3). The

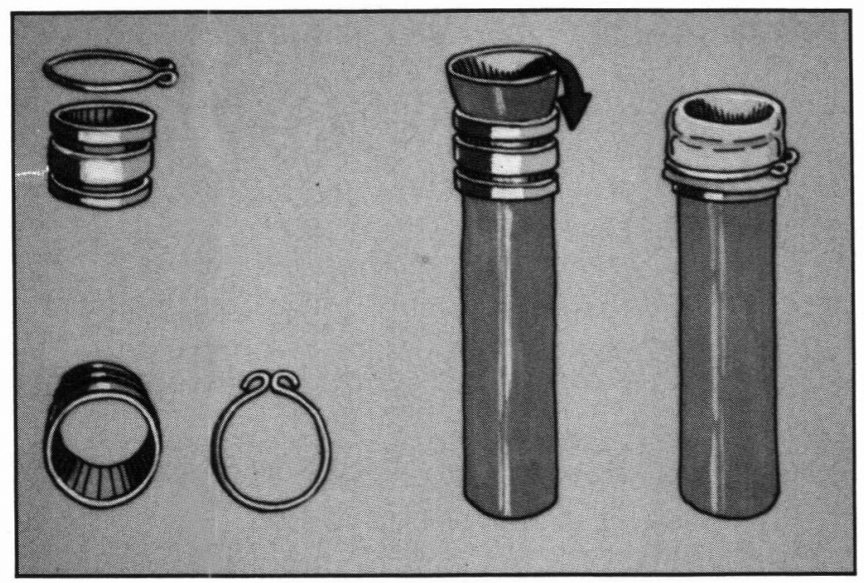

Figure 1 - Drawing of devicefor end-to-endnon-suture anastomosis, demonstrating the grooved cylinder and ring-clip.

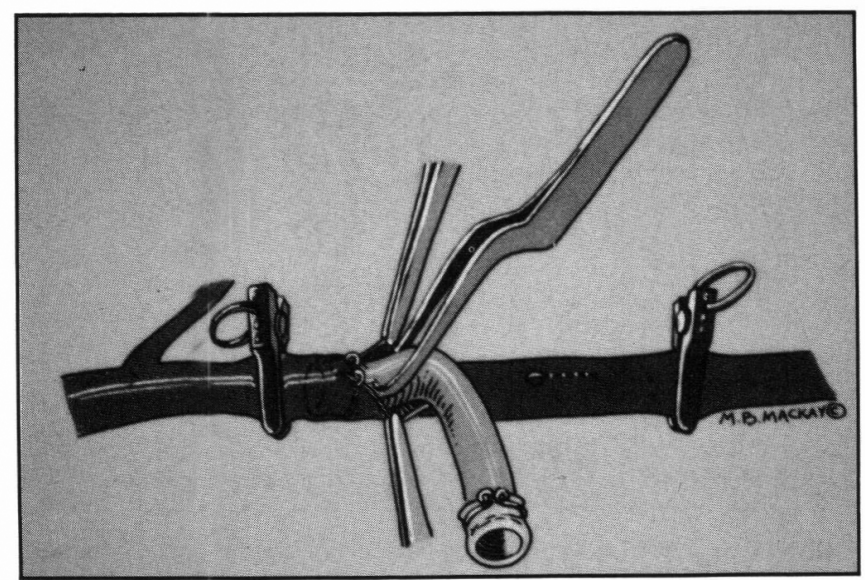

Figure 2 - Drawing of non-suture, end-to-end anastomosis using vein graft. segment of recipient artery chosen for the site of anastomosis is then prepared and temporarily clamped. A cruciate arteriotomy incision is made in the recipient vessel and the spiked ring clip is held directly over the arteriotomy site. The edges of the incised recipient artery are brought through the ring and everted over the spikes of the clip. The donor vessel mounted on the cylinder and held by cylinder forceps is then inserted into the arteriotomy while the clip is opened slightly and then closed under spring tension onto the cylinder (Figure 3).

\section{Histological and Biomechanical Studies}

Animals have been followed from I hour to I year postoperatively and patency rates documented with serial angiography. In-vivo patency tests were also performed prior to harvesting. The anastomotic site was excised and placed in 10\% buffered formalin for histological evaluation. After fixation, the metal cylinder and clips were removed and the vessel specimen dehy. drated and embedded in paraffin. Histological processing included longitudinal sectioning and staining with hematoxylin and eosin and elastin trichrome stain. The vessels were also processed for scanning electron microscopic studies.

The biomechanical properties of the non-suture anastomosis were assessed using an Instron unit (Instron Corporation, Canton. Massachusetts, U.S.A.), and the strength of the anastomosis was compared to standard suture technique. For this test, both ends of the segment of vessel containing either the non-suture or suture anastomosis were mounted on blunt needles and secured tightly. The vessels were inflated with $.9 \%$ saline solution with blue dye to detect fluid leakage during stress testing. A constant injection rate was applied until the vessel pulled apart. The load enlongation curve was plotted by strip chart recorder; non-suture and suture anastomoses were tested.

\section{RESULTS}

To date, over 400 non-suture anastomoses, which include interposition vein grafts, have been carried out in rat. rabbit and dog vessels varying in size from $0.7 \mathrm{~mm}$ (rat femoral artery) to $4 \mathrm{~mm}$ (dog carotid artery) in diameter. The mean time for non-suture end-to-end anastomosis in our last 200 vessels have been $3.0 \pm 1.8 \mathrm{mins}$, compared with $15.2 \pm 3.8 \mathrm{mins}$ using the suture method and $5.5 \pm 2.2 \mathrm{mins}$ for non-suture end-to-side anastomosis, and $30.0 \pm 3.5 \mathrm{mins}$ for end-to-

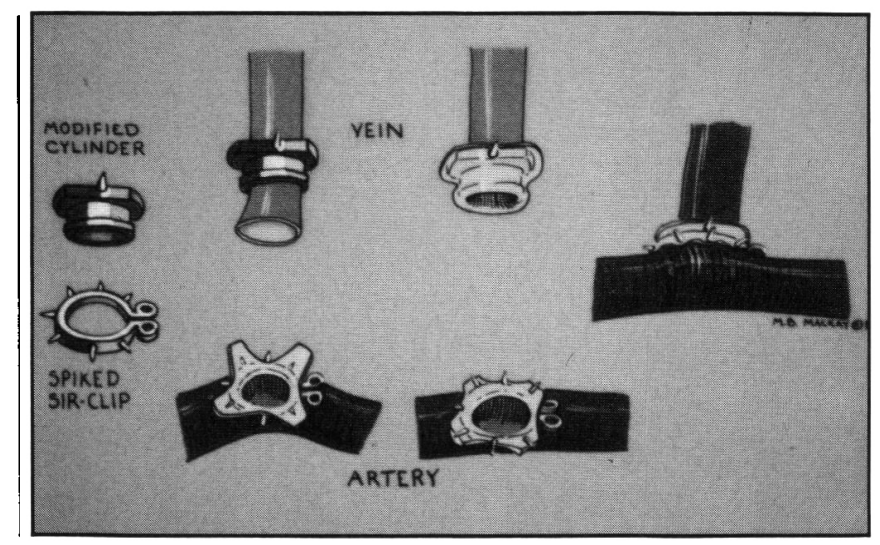

Figure 3 - Drawing of device and technique for end-1o-side non-sumure anastomosis. demonstrating the spiked cylinder and ring-clip. 
side anastomosis using standard suture technique; the time difference between the two methods is significant $(p<0.01)$. In a recent series of 124 non-suture anastomoses carried out using the rabbit carotid artery with external diameters ranging from $1.5 \mathrm{~mm}$ to $2.75 \mathrm{~mm}$, there was $100 \%$ patency rate for non-suture end-to-end anastomosis and $97.1 \%$ patency for end-to-side nonsuture anastomosis. ${ }^{2}$ Overall patency rates in non-suture, endto-end anastomosis using an interposition vein graft was $85 \%$ at 1 year follow up. The patency rates ranged from $76 \%$ in $2 \mathrm{~mm}$ vessels to $100 \%$ in $4 \mathrm{~mm}$ vessels. Figure 4 is an angiogram of a dog's carotid arteries I year following non-suture anastomosis using bilateral interposition vein grafts; the diameter of the arteries was $3 \mathrm{~mm}$; the anastomoses remain patent with no evidence of narrowing of the anastomotic sites. Figure 5 is an angiogram of an interposition vein graft in a dog's brachial vessel 6 months postoperatively, showing it to be widely patent.

Histological analysis revealed a healing process at the nonsuture anastomotic site temporally similar to suture anastomosis. While significant damage to endothelium and platelet fibrin microthromboses were invariably seen at the suture anastomotic site in the early period following surgery, related to needle suture trauma, none was found at the non-suture site. The process of re-endothelialization as seen on scanning electron microscopy was similar in both suture and non-suture anastomosis and usually complete by 2 to 3 weeks. Figure 6 is a

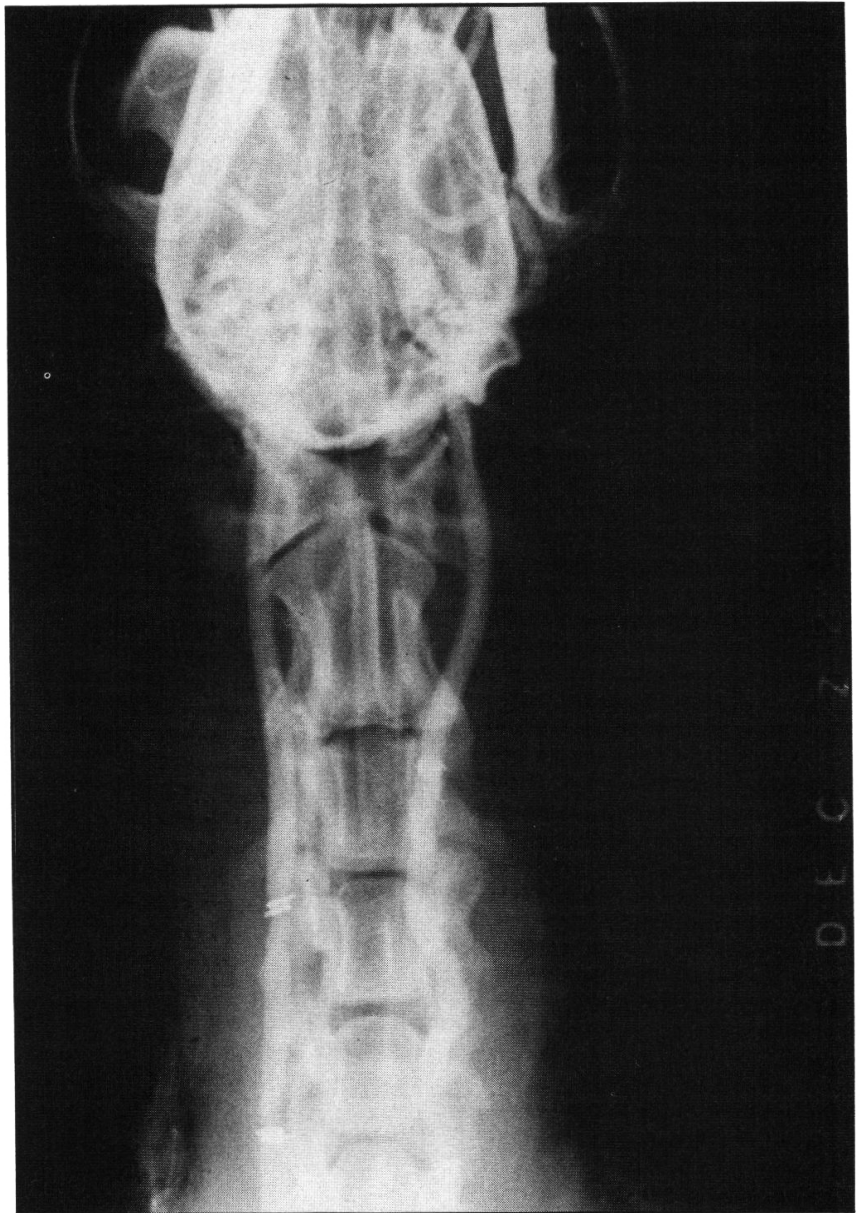

Figure 4-Angiogram of dog carotid vessels showing patent bilateral interposition vein grafts (Rt, 6 months, Lt. 1 year postoperatively). photo micrograph of a non-suture anastomosis 2 months following surgery. Examination of the portion of vessel that had been within the anastomotic cylinder revealed a degree of thinning first evident at 3 months, and which stabilized at approximately 6 months. This involved primarily the media and adventitia. In all cases the integrity of the intima was maintained. The degree of attenuation at 1 year was similar to that at 6 months. Aneurysmal formation was not encountered with the non-suture technique. The mean burst and disruptive strength in the non-suture anastomosis was similar to that of the suture technique and by 6 weeks compared favourably with normal vessel strength (Figure 7).

\section{Studies in Human Cadaver Vessels}

A number of studies of fresh human cervical and intracranial cadaver vessels have also been carried out to obtain information such as vessel wall thickness and other parameters that

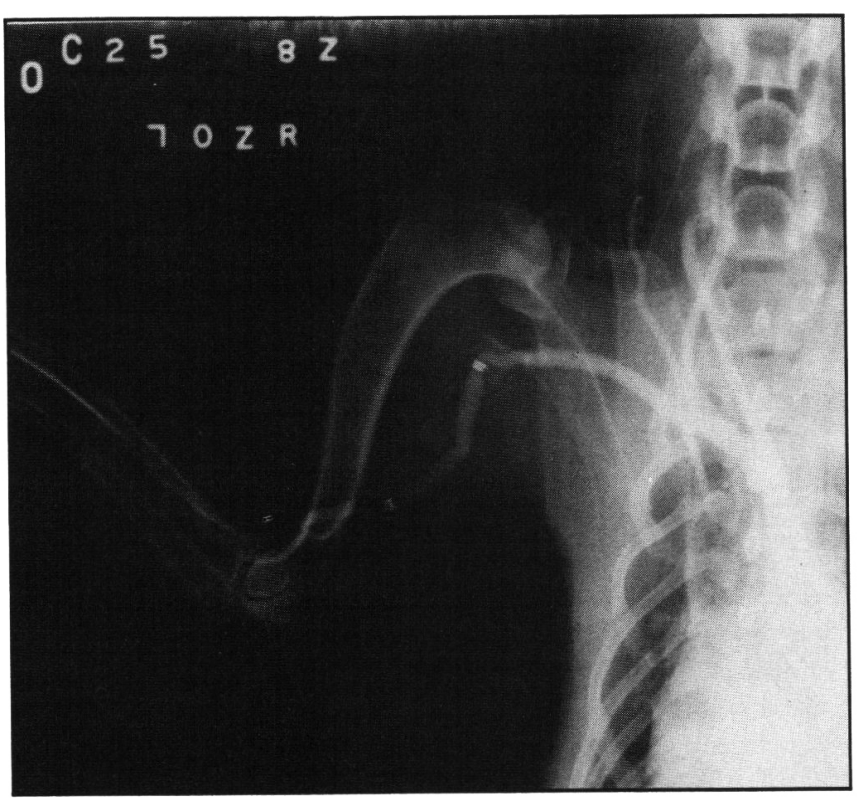

Figure 5 - Angiogram showing patent interposition vein graft to $2.0 \mathrm{~mm}$ brachial vessel in dog 6 months postoperatively.

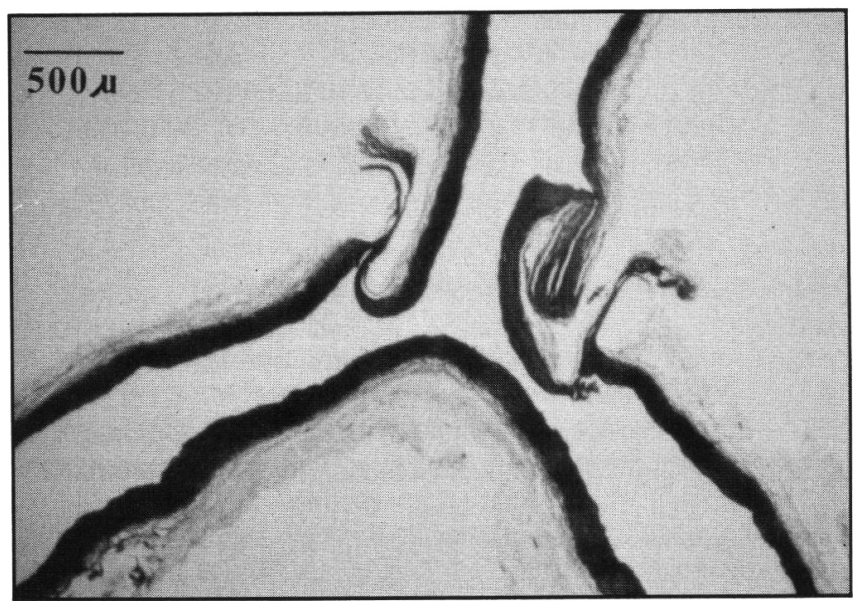

Figure 6 - Photomicrograph of non-suture, end-to-sideanastomosis 2 months postoperatively. Metal components of the prosthesis have been removed. Elastin trichrome stain. Original magnification: $x 20$. 


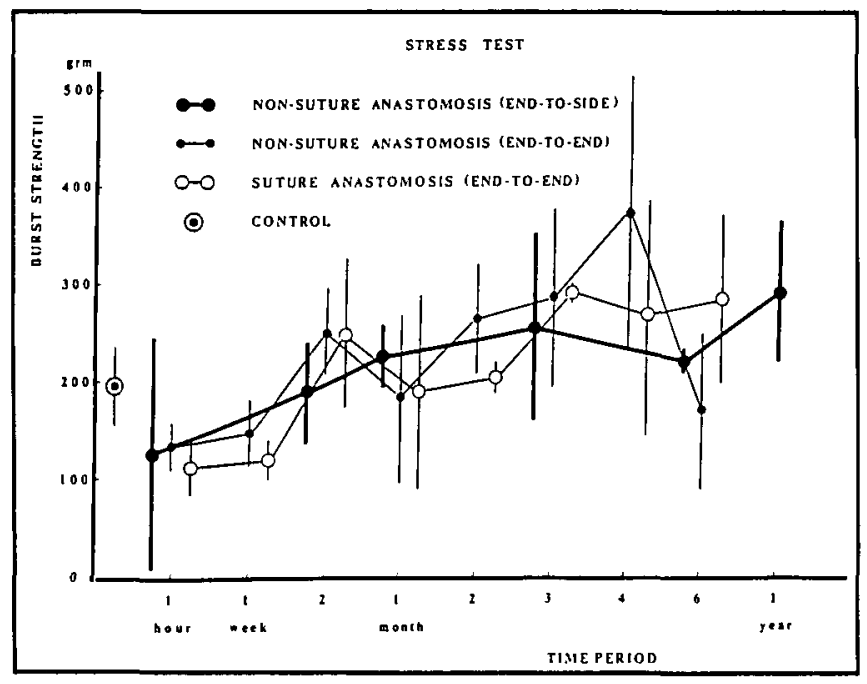

Figure 7 - Graph of burst strength measurements of suture $(n=60)$, and non-suture $(n=124)$ anastomosis in rabbit carotid artery.

would be required in human application of the technique. Cadaver studies suggest that the technique could be applied favourably to intracranial internal carotid and $M 1, M 2$, and $M 3$ segments of the middle cerebral artery. A segment of vessel free of atherosclerotic plaque would be required for application of the end-toside prosthesis.

\section{Discussion}

There have been many attempts over the years to develop alternative techniques for vascular anastomosis. ${ }^{3-11}$ The potential advantages of a non-suture technique would include reduction of operating time and the elimination of suture material within the vessel lumen, with reduced initimal trauma and the potential for thrombus formation. For a technique to become widely acceptable, it must be relatively simple. The method should be applicable to a variety of human vessel sizes, and should rival or better the success rates using suture techniques. Despite all efforts, however, the results of non-suture techniques have generally been poor and, to date, none have come into common clinical use.

Our previous studies provided validity to the concept of a non-suture vascular anastomosis. ${ }^{\prime}$ The favourable patency rates would appear to be related to precise alignment and intima-tointima opposition at the site of anastomosis without the presence of suture material within the lumen. Histological and biomechanical studies show that our technique compares favourably with conventional suture anastomosis. There was no significant increase in tissue reaction in or around the non- suture anastomotic site, a fact attributable to the inert and biocompatible materials from which the prosthesis is fashioned. There was some degree of attentuation of the vessel wall at the site of the anastomotic cylinder; this stabilized at 6 months and was not associated, on biomechanical evaluation, with weakening of the anastomosis. The results of these studies together with our studies using fresh human cadaver vessels have suggested to us the technical feasibility of using this technique in man. Clinical application of non-suture technique is currently underway. In neurosurgery, the technique will be useful in the treatment of inaccessible regional stenotic lesions of both anterior and posterior circulation, and in dealing with large complex aneurysms where one can rapidly and fully eliminate the aneurysm from the circulation. It is felt, however, that this method may have an even greater role in cardiac, peripheral vascular, transplantation and re-implantation surgery.

\section{ACKNOWLEDGEMENT}

The assistance of David Walsh is gratefully acknowledged. The work has been supported by grants from Idea Corporation. Ontario. and C.D.C. Imasco Corporation. Canada.

\section{REFERENCES}

1. Lougheed WM. Gentili F. Fazl M. MacKay MB. A non-suture technique for rapid vascular anastomosis. In: Cerebral Revascularization for Stroke. Spetzler RF \& Carter LP. eds: ThiemeStratton. New York, 1985: 243-248.

2. Yoshijima S. Gentili F, Lougheed WM, et al. A comparison of suture and non-suture vascular anastomosis - Biomechanical and histological evaluation. Proceedings of 8 th International Symposium on Microvascular Anastomoses for Cerebral Ischaemia (in press)

3. Weiss EW. Lam CR. Tantalum tubes in non-suture method of blood vessel anastomosis. Am J Surg 1950; 80: 452-454.

4. Inokuchi K. A new type of blood vessel suturing apparatus. Arch Surg 1958; 80: 452-454.

5. Nakayama K. Tamiya T, Yamamoto K, et al. A simple new apparatus for small vessel anastomosis (free autografts of sigmoid included). Surgery 1962. 52: 918-931.

6. Andrrosov PI. New method of surgical treatment of blood vessel lesions. Arch Surg 1956: 73: 902-910.

7. Ruckland D, Schumacher PH, Mueller KM, et al. Experimental studies with a new sutureless anastomotic flange. Trans Am Soc Artif Intern Organs 1979: 25: 339-343.

8. Yamagata $S$, Handa $H$, Taki $W$. et al. Experimental nonsuture microvascular anastomosis using a soluble PVA tube and plastic adhesive. J Microsurg 1979: 1: 208-215.

9. Kewel K. Sutureless microvascular anastomosis using a neodymiumYAG laser. J Microsurg 1980: 1: 436-439.

10. Wintermantel $\mathbf{E}$. The thermic vascular anastomosis. A new nonsuture method. Acta Neurochir 1981: 56: 5-24.

11. Daniell RK, Olding M. An absorbable anastomotic device for microvascular surgery: Experimental studies. Reconst Surg 1984: 74: 329-336. 